\title{
Robust active contour segmentation with an efficient global optimizer
}

\author{
Jonas De Vylder, Jan Aelterman, and Wilfried Philips \\ Department of Telecommunications and Information Processing, \\ IBBT - Image Processing and Interpretation, \\ Ghent University, St-Pietersnieuwstraat 41, B-9000 Ghent, Belgium \\ jonas.devylderetelin.ugent.be \\ http://telin.ugent.be/ jdvylder/
}

\begin{abstract}
Active contours or snakes are widely used for segmentation and tracking. Recently a new active contour model was proposed, combining edge and region information. The method has a convex energy function, thus becoming invariant to the initialization of the active contour. This method is promising, but has no regularization term. Therefore segmentation results of this method are highly dependent of the quality of the images. We propose a new active contour model which also uses region and edge information, but which has an extra regularization term. This work provides an efficient optimization scheme based on Split Bregman for the proposed active contour method. It is experimentally shown that the proposed method has significant better results in the presence of noise and clutter.
\end{abstract}

Key words: Active contours, segmentation, convex optimization, Split Bregman

\section{Introduction}

Since Kass et al. [1] introduced there snakes, the active contour framework has become a constant recurring topic in segmentation literature. The framework allows easy tuning to specific segmentation and tracking problems. Prior motion information of objects which need to be tracked [2-4], specific shape models [2,5,6], region statistics of objects $[7,8]$, etc. These are just a small notion of different forms of prior knowledge which have been incorporated in the active contour framework.

In the active contour framework, an initial contour is moved and deformed in order to minimize a specific energy function. This energy function should be minimal when the contour is delineating the object of interest, e.g. a leaf. Two main groups can be distinguished in the active contour framework: one group representing the active contour explicitly as a parameterized curve and a second group which represents the contour implicitly using level sets. In the first group, also called snakes, the contour generally converges towards edges in the image $[1,9,5]$. The second group generally has an energy function based on region properties, such as variance of intensity of the enclosed segment $[7,10]$. These level set approaches has gained a lot of interest since they have some benefits over snakes. For example, they can easily change their topology, e.g. splitting a segment into multiple unconnected segments. Recently an active 
contour model has been proposed with a convex energy function, making it possible to define fast global optimizers $[11,12]$. These global active contours have the benefit that there result is no longer dependent on the initialization.

In [13], Bresson et al. proposed a new type of active contour with a convex energy function, a model which combined edge information and region information. This method combines the original snake model [1] with the active contour model without edges [7]. The proposed model has an energy function which is completely defined by the image, thus eliminating the possibility of regularization. Although the method has some interesting benefits, it lacks robustness to noise and clutter. To tackle this problem, we propose a new active contour model which has the benefits of the model proposed in [13], but which has an extra regularization term. This regularization term enforces smoothness of the boundaries of the segments. This results in segments with a smooth boundary, i.e. avoiding jaggy edges due to noise.

This paper is arranged as follows. The next section briefly enumerates the notations and symbols used in this paper. In section 3 the current state of the art of global optimum active contours is summarized and expanded with the proposed method. Section 4 elaborates on a fast optimization method which can be used to calculate the proposed active contour. The next section shows some examples and quantitative results of our technique in comparison to other active contour formulations. Both convergence speed and segmentation result are examined. Section 6 recapitulates and concludes.

\section{Notations and Definitions}

In the remaining of this paper we will use specific notations some conventional, some more peculiar to this work. Therefore we briefly summarize the notations and symbols used in this work.

We will refer to an image, $F$ in its vector notation, i.e. $\mathbf{f}(i * m+j)=F(i, j)$, where $m \times n$ is the dimension of the image. In a similar way we will represent the contour in vector format, $\mathbf{u}$. If a pixel $U(i, j)$ is part of the segment, it will have a value above a certain threshold, all background pixels will have a value lower than the given threshold. Note that this is similar to level-sets. The way these contours are optimized however is different than with classical level-set active contours, as is explained in the next section. We will use image operators, i.e. gradient, divergence and Laplacian in combination with this vector notation, however the semantics of the image operators remains the same as if it was used with the classical matrix notation:

$$
\begin{aligned}
\nabla(\mathbf{f}(i * m+j)) & =(F(i+1, J)-F(i, j), F(i, J+1)-F(i, j)) \\
\nabla \cdot(\mathbf{f}(i * m+j)) & =(F(i+1, J)-F(i, j))+(F(i, J+1)-F(i, j)) \\
\nabla^{2}(\mathbf{f}(i * m+j)) & =\nabla \cdot \nabla(\mathbf{f}(i * m+j))
\end{aligned}
$$


Further we will use the following inner product and norm notations:

$$
\begin{aligned}
\langle\mathbf{f}, \mathbf{g}\rangle & =\sum_{i=1}^{m n} f(i), g(i) \\
\|\mathbf{f}\|_{\alpha, \mathbf{g}} & =\left(\sum_{i=1}^{m n} g(i)(|f(i)|)^{\alpha}\right)^{\frac{1}{\alpha}}
\end{aligned}
$$

If the weights $g(i)=1$ for all $i$, then we will omit $\mathbf{g}$, since we assume this will not cause confusion, but will increase readability.

\section{Convex Energy Active Contours}

In [11] an active contour model was proposed which has global minimizers. This active contour is calculated by minimizing the following convex energy:

$$
E[\mathbf{u}]=\|\nabla \mathbf{u}\|_{1}+\mu\langle\mathbf{u}, \mathbf{r}\rangle
$$

with

$$
\mathbf{r}=(m f-\mathbf{f})^{2}-(m b-\mathbf{f})^{2}
$$

Here $\mathbf{f}$ represents the intensity values in the image, $m f$ and $m b$ are respectively the mean intensity of the segment and the mean intensity of the background, i.e. every pixel not belonging to the segment. Note that this energy is convex, only if $m f$ and $m b$ are constant. This problem can be solved by iterating between the following two steps: first fix $m f$ and $m b$ and minimize eq. (1), secondly update $m f$ and $m b$. Chan et al. found that the steady state of the gradient flow corresponding to this energy, i.e.

$$
\frac{d \mathbf{u}}{d t}=\nabla \cdot \frac{\nabla \mathbf{u}}{|\nabla \mathbf{u}|}-\mu \mathbf{r}
$$

coincides with the steady state of the gradient flow of the original Chan-Vese active contours [11,7]. So minimizing eq. (1) is equivalent to finding an optimal contour which optimizes the original Chan-Vese energy function. Although the energy in eq. 1 does not have a unique global minimizer, a well defined minimizer can be found within the interval $[0,1]^{n}$ :

$$
\mathbf{u}^{*}=\underset{\mathbf{u} \in[0,1]^{n}}{\arg \min }\|\nabla \mathbf{u}\|_{1}+\mu\langle\mathbf{u}, \mathbf{r}\rangle
$$

Note that this results in a minimizer which values are between 0 and 1 . It is however desirable to have a segmentation result where the values of a minimizer are constrained to $(0,1)$, i.e. a pixel belongs to a segment or not. Therefore $\mathbf{u}^{*}$ is tresholded, i.e.

$$
\Phi_{\alpha}\left(u^{*}(x)\right)= \begin{cases}1 & \text { if } u^{*}(x)>\alpha \\ 0 & \text { otherwise }\end{cases}
$$

with a predefined $\alpha \in[0,1]$. In [14] it is shown that $\Phi_{\alpha}\left(\mathbf{u}^{*}\right)$ is a global minimizer for the energy in eq. (1) and by extension for the energy function of the original Chan-Vese 
active contour model. In [13] the convex energy function in eq. (1) was generalized in order to incorporate edge information:

$$
E[\mathbf{u}]=\|\nabla \mathbf{u}\|_{\mathbf{1}, \mathbf{g}}+\mu\langle\mathbf{u}, \mathbf{r}\rangle
$$

where $\mathrm{g}$ is the result of an edge detector, e.g. $\mathrm{g}=\frac{1}{1+|\nabla \mathbf{f}|}$. The active contour minimizing this energy function can be seen as a combination of edge based snake active contours [1] and the region based Chan-Vese active contours [7]. Since this method only minimizes energy terms based on the image, it is highly influenced by the quality of the image. In the presence of noise and clutter the method will find false segments or distorted segment boundaries. In order to make the method more robust we propose to extend the energy function in eq. (6) with an extra regularization term:

$$
E[\mathbf{u}]=\|\nabla \mathbf{u}\|_{1}+\gamma\|\nabla \mathbf{u}\|_{1, \mathbf{g}}+\mu\langle\mathbf{u}, \mathbf{r}\rangle
$$

Where $\gamma$ is a weighting parameter defining the influence of the extra regularization therm. This regularization term approximates the length of the segments boundary, thus penalizing small false segments and high curved boundaries due to noise.

\section{Optimization}

Due to the convexity of the energy function in eq. (7), a wide range of minimizers can be used to find an optimal contour $\mathbf{u}^{\dagger}$. The Split Bregman method is an efficient optimization technique for solving L1-regularized problems and has good convergence properties. In order to find a contour which minimizes eq. (7), the Split Bregman method will "de-couple" the L1 and L2 norm, by introducing a new variable $\mathbf{d}$ and by putting constraints on the problem. This results in the following optimization problem:

$$
\left(\mathbf{u}^{\dagger}, \mathbf{d}^{\dagger}\right)=\underset{\mathbf{u}, \mathbf{d}}{\arg \min }\|\mathbf{d}\|_{1}+\gamma\|\mathbf{d}\|_{1, \mathbf{g}}+\mu\langle\mathbf{u}, \mathbf{r}\rangle \text { such that } \mathbf{d}=\nabla \mathbf{u}
$$

This optimization problem can be converted to an unconstrained problem by adding a quadratic penalty function, i.e.

$$
\left(\mathbf{u}^{\dagger}, \mathbf{d}^{\dagger}\right)=\underset{\mathbf{u}, \mathbf{d}}{\arg \min }\|\mathbf{d}\|_{1}+\gamma\|\mathbf{d}\|_{1, \mathbf{g}}+\mu\langle\mathbf{u}, \mathbf{r}\rangle+\frac{\lambda}{2}\|\mathbf{d}-\nabla \mathbf{u}\|_{2}^{2}
$$

Where $\lambda$ is a weighting parameter. If $\gamma$ is heigh, $\mathbf{d}=\nabla \mathbf{u}$. However setting $\gamma$ high introduces numerical instability. Note that the quadratic penalty function only approximates the constraint $\mathbf{d}=\nabla \mathbf{u}$. However, by using a Bregman iteration technique [15], this constraint can be enforced exactly in an efficient way. In the Bregman iteration technique an extra vector, $\mathbf{b}_{\mathbf{k}}$ is added to the penalty function. Then the following two unconstrained steps are iteratively solved.

$$
\begin{aligned}
\left(\mathbf{u}_{\mathbf{k}+\mathbf{1}}, \mathbf{d}_{\mathbf{k}+\mathbf{1}}\right) & \left.=\underset{\mathbf{u}_{\mathbf{k}}, \mathbf{d}_{\mathbf{k}}}{\arg \min }\left\|\mathbf{d}_{\mathbf{k}}\right\|_{1}+\gamma\left\|\mathbf{d}_{\mathbf{k}}\right\|_{1, \mathbf{g}}+\mu\left\langle\mathbf{u}_{\mathbf{k}}, \mathbf{r}\right\rangle+\frac{\lambda}{2}\left\|\mathbf{d}_{\mathbf{k}}-\nabla \mathbf{u}_{\mathbf{k}}-\mathbf{b}_{\mathbf{k}}\right\| \|_{2} 0\right) \\
\mathbf{b}_{\mathbf{k}+\mathbf{1}} & =\mathbf{b}_{\mathbf{k}}+\nabla \mathbf{u}_{\mathbf{k}+\mathbf{1}}-\mathbf{d}_{\mathbf{k}+\mathbf{1}}
\end{aligned}
$$


The first step requires optimizing for two different vectors. We approximate these optimal vectors by alternating between optimizing eq. (10) for $\mathbf{u}$ and optimizing eq. (10) for $\mathbf{d}$ independently:

$$
\begin{aligned}
& \mathbf{u}_{\mathbf{k}+\mathbf{1}}=\underset{\mathbf{u}_{\mathbf{k}}}{\arg \min } \mu\left\langle\mathbf{u}_{\mathbf{k}}, \mathbf{r}\right\rangle+\frac{\lambda}{2}\left\|\mathbf{d}_{\mathbf{k}}-\nabla \mathbf{u}_{\mathbf{k}}-\mathbf{b}_{\mathbf{k}}\right\|_{2}^{2} \\
& \mathbf{d}_{\mathbf{k}+\mathbf{1}}=\underset{\mathbf{d}_{\mathbf{k}}}{\arg \min }\left\|\mathbf{d}_{\mathbf{k}}\right\|_{1}+\gamma\left\|\mathbf{d}_{\mathbf{k}}\right\|_{1, \mathbf{g}}+\frac{\lambda}{2}\left\|\mathbf{d}_{\mathbf{k}}-\nabla \mathbf{u}_{\mathbf{k}+\mathbf{1}}-\mathbf{b}_{\mathbf{k}}\right\|_{2}^{2}
\end{aligned}
$$

The first problem can be optimized by solving a set of Euler-Lagrange equations. For each element $u(i)$ of the optimal $\mathbf{u}$ the following optimality condition should be satisfied:

$$
\nabla^{2} u(i)=\frac{\mu}{\lambda} r(i)+\nabla \cdot(\mathbf{d}(i)-\mathbf{b}(i))
$$

Note that this system of equations can be written as $A \mathbf{u}=\mathbf{w}$. In [12] they proposed to solve this linear system using the iterative Gauss-Seidel method. In order to guarantee the convergence of this method, $A$ should be strictly diagonally dominant or should be positive semi definite. Unfortunately is $A$ neither. Instead we will optimize eq. (14) using the iterative conjugate residual method, which is a Krylov subspace method for which convergence is guaranteed if $A$ is Hermitian [16].

The solution of eq. (14) is unconstrained, i.e. $u(i)$ does not have to lie in the interval $[0,1]$. Note that minimizing eq. (12) for $u(i)$, i.e. all other elements of $u$ remain constant, is equivalent to minimize a quadratic function. If $u(i) \notin[0,1]$ then the constrained optimum is either 0 or 1 , since a quadratic function is monotonic in an interval which does not contain its extremum. So the constrained optimum can be calculated as follows:

$$
u^{*}(i)=\max (\min (u(i), 1), 0)
$$

In order to calculate an optimal $\mathbf{d}_{\mathbf{k}}$, we can rewrite eq. (13) as follows:

$$
\mathbf{d}_{\mathbf{k}+\mathbf{1}}=\underset{\mathbf{d}_{\mathbf{k}}}{\arg \min }\left\|\mathbf{d}_{\mathbf{k}}\right\|_{1,(\mathbf{1}+\gamma \mathbf{g})}+\frac{\lambda}{2}\left\|\mathbf{d}_{\mathbf{k}}-\nabla \mathbf{u}_{\mathbf{k}+\mathbf{1}}-\mathbf{b}_{\mathbf{k}}\right\|_{2}^{2}
$$

A closed form solution for this optimization step can be calculated using the shrinking operator, i.e.

$$
\mathbf{d}_{\mathbf{k}+\mathbf{1}}(i)=\operatorname{shrink}\left(\nabla \mathbf{u}(i)+\mathbf{b}_{\mathbf{k}}, 1+\gamma g(i), \lambda\right)
$$

where

$$
\operatorname{shrink}(\tau, \theta, \lambda)= \begin{cases}0 & \text { if }\|\tau\| \leq \frac{\theta}{\lambda} \\ \tau-\frac{\theta}{\lambda} \operatorname{sgn}(\tau) & \text { otherwise }\end{cases}
$$

In algorithm 1 we give an overview in pseudo code of the complete optimization algorithm. As an initial value for $\mathbf{d}[t]$ and $\mathbf{b}[t]$ we chose $(0,0)$. The initial estimation of $m f$ and $m b$ can be calculated based on Otsu thresholding. The CR function solves eq. (14) using the Conjugate residual method, given the parameters $\mathbf{b}_{\mathbf{k}}, \mathbf{d}_{\mathbf{k}}$ and $\mathbf{r}_{\mathbf{k}}$. Note that the last line is the update of $\mathbf{r}_{\mathbf{k}}$ based on the new mean intensity of the foreground/background, which were calculated in the previous two lines of code. 


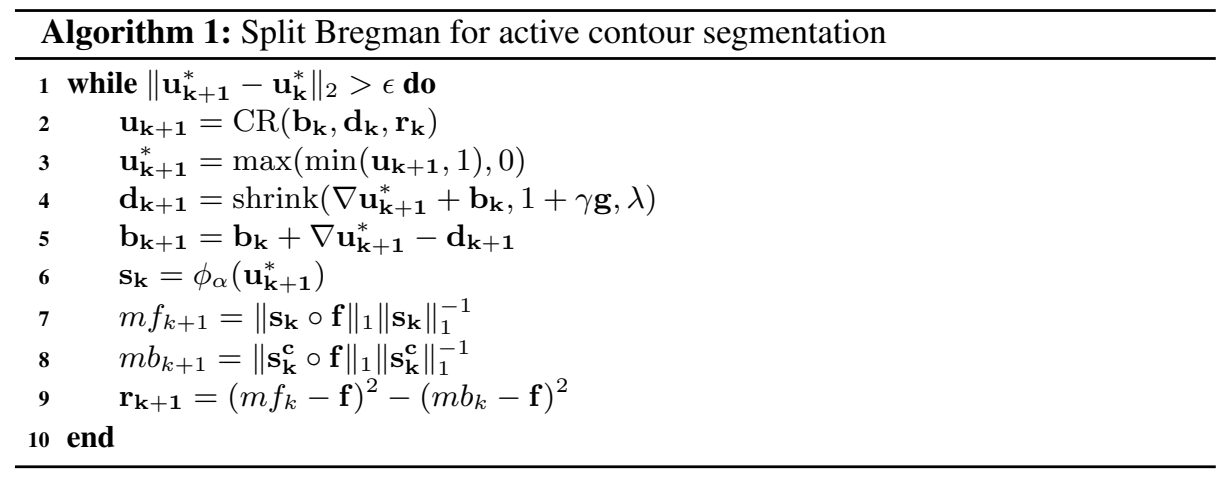

\section{Results}

\subsection{Examples}

A typical application for active contours is segmentation of organs in medical images. As an example Fig. 1 shows the result of segmenting white matter in an MRI image of the brain. The top row shows the RAW MRI image on the left and the segmentation result of the Chan-Vese convex active contour (CVAC); this uses regularization but does not incorporate edge information. The CVAC shows a good segmentation result, although the method does make some small errors near the borders of the contour. These errors generally exists of background pixels with "high" intensity which are considered to be foreground. Due to their "high" intensity they resemble the segment, however considering the neighbouring edges; it is unlikely that they actually belong to the segment, e.g. the white matter in this example. Some of these segmentation errors are indicated by the green arrows. The bottom row of Fig. 1fig:exBrain shows the segmentation results of CVAC which incorporate edge information. The left image does not incorporate a regularization term in its energy function, resulting in lots of small segments due to noise. The right image shows the segmentation result of the proposed active contour model. This active contour does not suffer from noise, nor has it the small errors near borders which occur with the original Chan-Vese active contour model.

A second example is shown in Fig: 2, where a squirrel has to be segmented out of a gray-scale image. The original CVAC, shown in the top row on the right, results in poor segmentation. A part of the head and a piece of the paw are missing in the segmentation result. Incorporating edge information helps to recover these missing parts as can be seen in the bottom row. Due to the clutter in the background the method finds a lot of false segments if there is no regularization, as can be seen on the left. The proposed method however finds the biggest part of the squirrel without adding any background pixels as is shown on the right of Fig. 2. Although the proposed method gives better results in noise images or images with noise, it comes with a cost, i.e. the active contour converges slower. The proposed method does converge slower than the CVAC which incorporates edge information. The convergence speed depends on the amount of regularization needed. If hardly any regularization is needed, i.e. $\gamma$ in eq. (7) $>>1$, the speed approaches the convergence speed of CVAC with edge information [13]. Whereas 

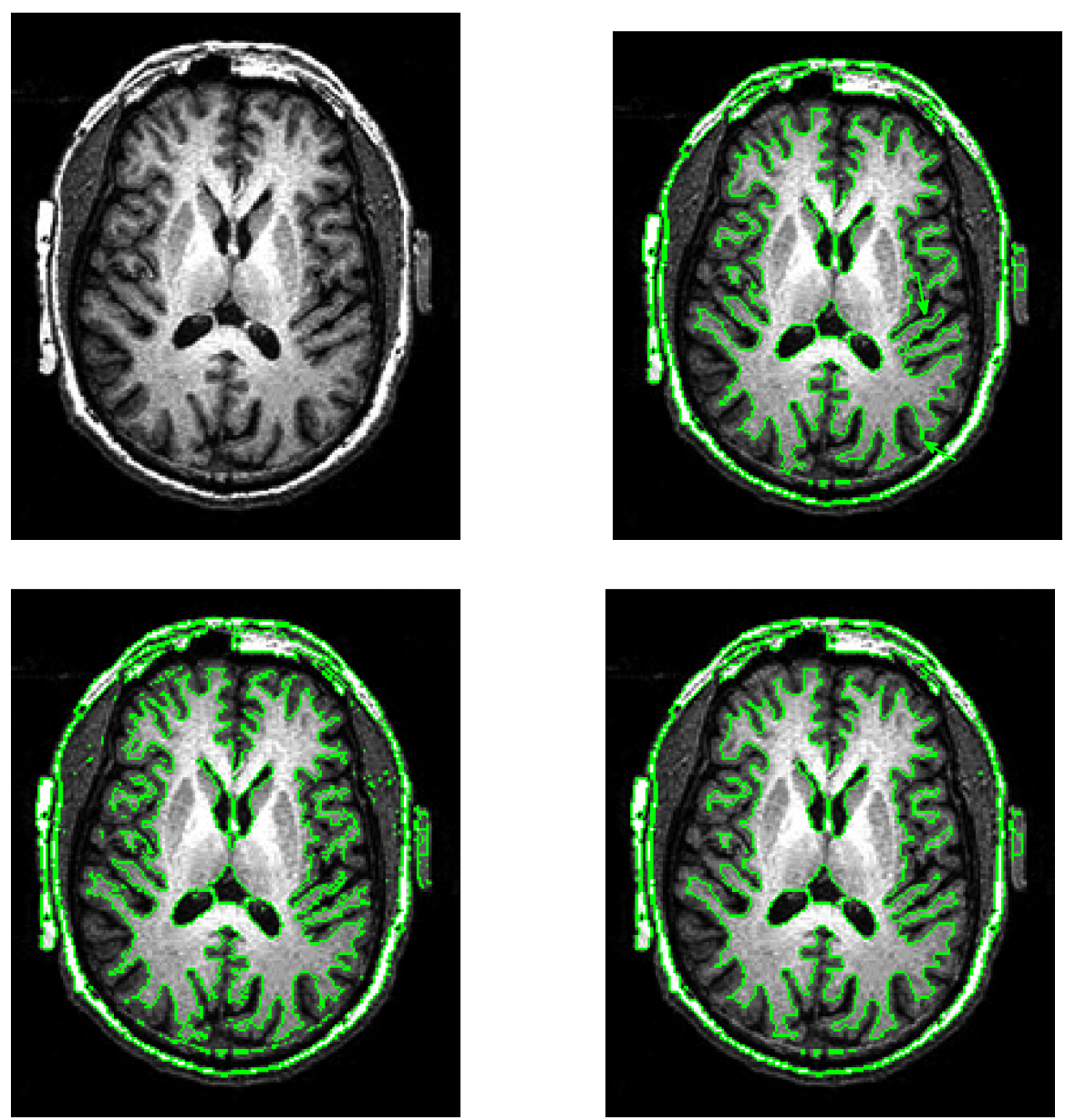

Fig. 1. An example of brain white matter segmentation in MRI images. Top left shows an MRI slice of the brain. Top right depicts the segmentation result of the Chan-Vese convex active contours. Bottom left shows the Chan-Vese convex active contour segmentation using edge information. Bottom right depicts the proposed segmentation method, i.e. active contours using edge information with regularization. 

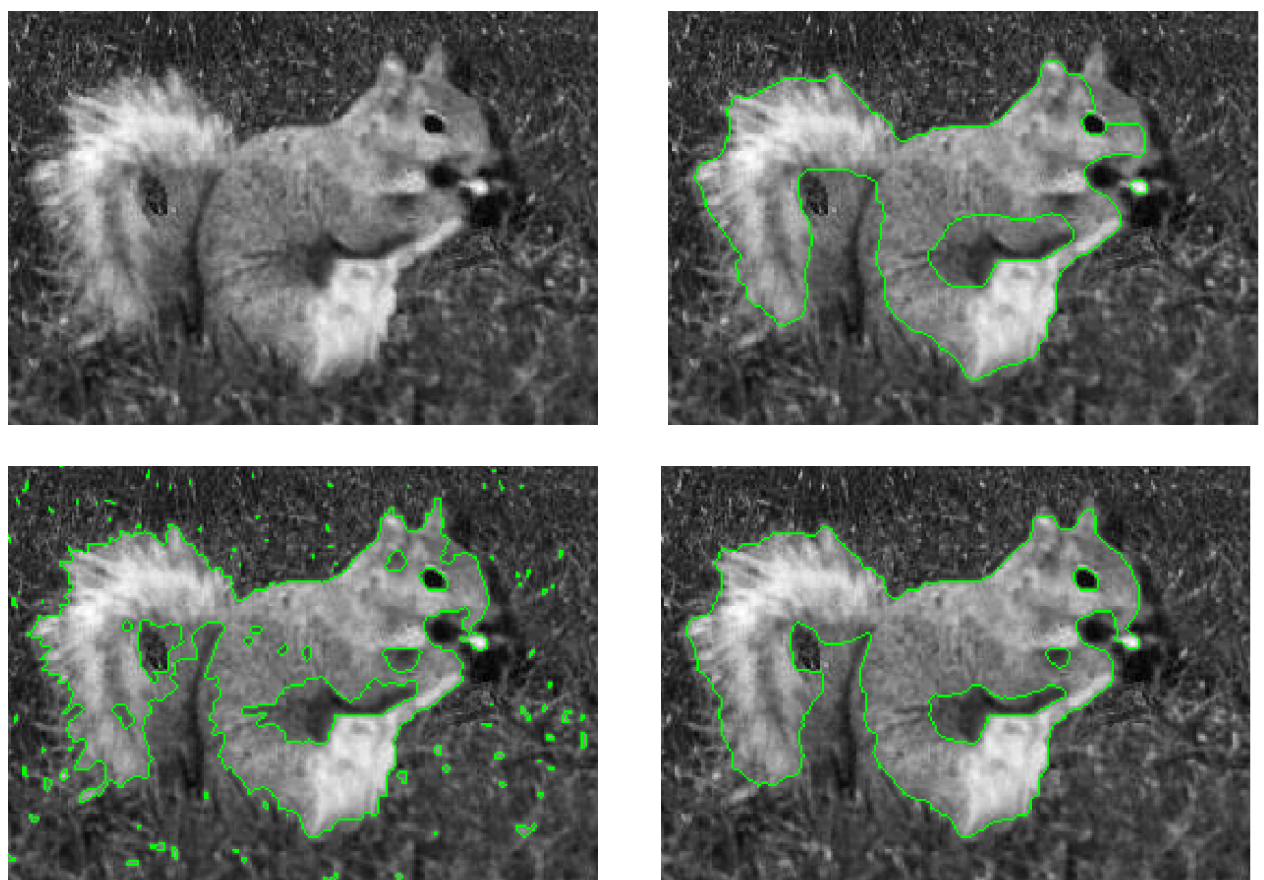

Fig. 2. An example of segmentation in photos with clutter. Top left shows a gray-scale photo of a squirrel. Top right depicts the segmentation result of the Chan-Vese convex active contours. Bottom left shows the Chan-Vese convex active contour segmentation using edge information. Bottom right depicts the proposed segmentation method, i.e. active contours using edge information with regularization. 


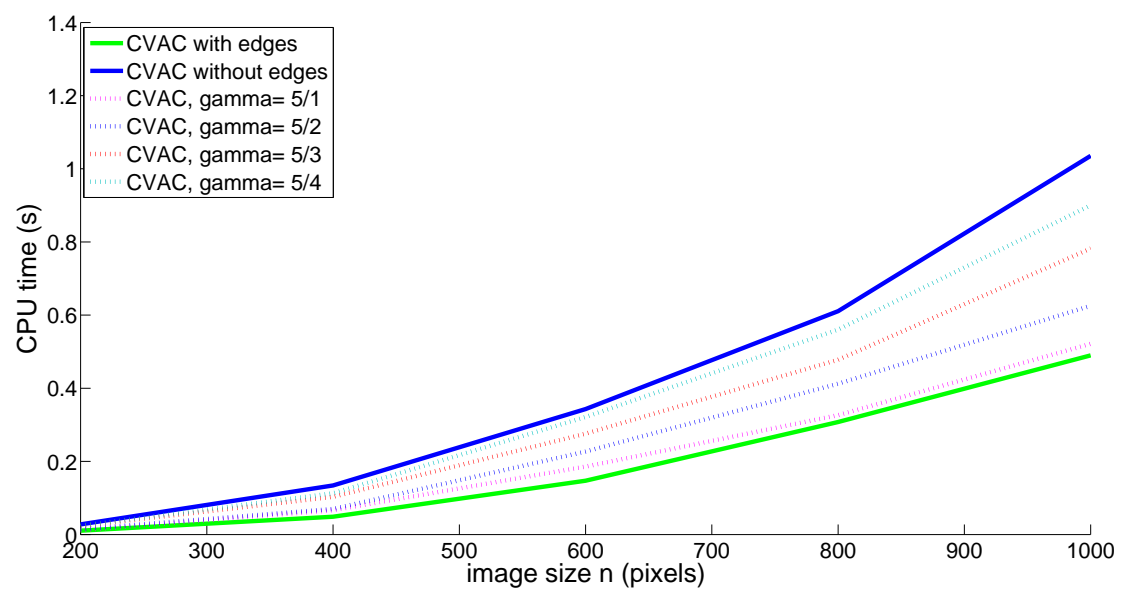

Fig. 3. Convergence speed of different active contours in function of the image size. The full lines are active contours from literature, Chan-Vese active contours with and without incorporation of edge information. The dotted lines represent the convergence speed of the proposed method with different $\gamma$, i.e. different ratio's of regulation.

if the regularization factor is dominant in the energy function, the convergence speed approaches the convergence speed of the original CVAC using Split Bregman optimization [15]. Fig. 3 shows the convergence speed in function of the image size. Between these four different CVAC only the $\gamma$ parameter was changed, $\mu$ and $\lambda$ was constant between all methods, i.e. 0.001 and 0.5 respectively. The full lines depict the CVAC's from literature, in green the method using edge information, in blue the method using regularization without edge information. The dotted lines show the convergence speed of the proposed method for different $\gamma$ in eq. (7).

\subsection{Error Metric}

For the validation of the segmentation, the Dice coefficient is used. If $\mathrm{S}$ is the resulting segment from the active contour, i.e. $\phi_{0.5} \mathbf{u}^{*}$, and GT the ground truth segment based on manual segmentation, then the Dice coefficient between $\mathrm{S}$ and GT is defined as:

$$
d(S, G T)=\frac{2 \operatorname{Area}(S \wedge G T)}{\operatorname{Area}(\mathrm{S})+\operatorname{Area}(G T)}
$$

where $S \wedge G T$ consist of all pixels which both belong to the detected segment as well as to the ground truth segment. If S and GT are equal, the Dice coefficient is equal to one. The Dice coefficient will approach zero if the regions hardly overlap.

\subsection{Noise robustness}

In order to quantitatively validate the proposed method, a dataset of synthetic fluorescent microscopic images was segmented. This dataset has been developed by Ruusuvuri 

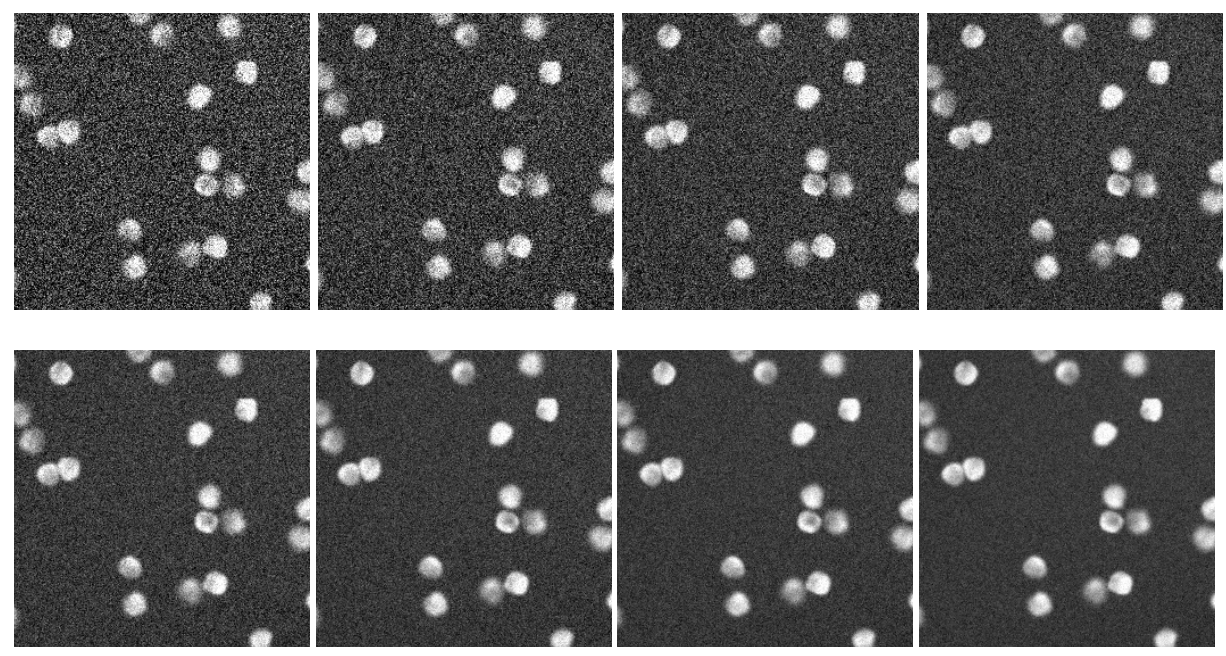

Fig. 4. An example of the micorscopy dataset used for vallidating the robustness of the proposed segmentation technique. The image is contaminated with different levels of white Gaussian noise. The top row has a SNR of 3,5,7 and 9 respectively. The bottom row as a SNR of 11,13,15 and 17 respectively.

et al. [17] and serves as a benchmark for segmentation algorithms. The dataset consists of 20 images each containing the 300 fluorescent nuclei. We contaminated this dataset with eight different levels of white Gaussian noise so that we could measure the influence of noise on the proposed segmentation technique. A close up of such an image with different noise levels can be seen in Fig. 4. The segmentation quality of the proposed method was measured using the average Dice coefficient for the full dataset and compared with the average Dice coefficient of segmentation using CVAC with edge information. As can be seen in Fig. 3 is the proposed method significantly more robust. The proposed method still gets a Dice coefficient of 0.7 for an image set with a SNR of only 3 in comparison with a Dice coefficient of 0.4 for the state of the art CVAC.

\section{Conclusion}

In this paper a new active contour method has been proposed. The method is comparable with the work proposed in [13], both methods are a combination of the original Chan-Vese active contours and snakes. The proposed method allows extra regularization, which was not possible in the method proposed by Bresson. The proposed method uses a convex energy function, allowing the use of global optimizers. This paper proposes a regularization term which enforces a smooth contour. However other convex regularization terms could be used as well, for example regularization using non local self similarity or based on texture such as is done in [18]. An efficient optimizer has been proposed using the Split Bregman optimization scheme. This results in efficient and fast optimization, although not as fast as the active contour proposed by Bresson 


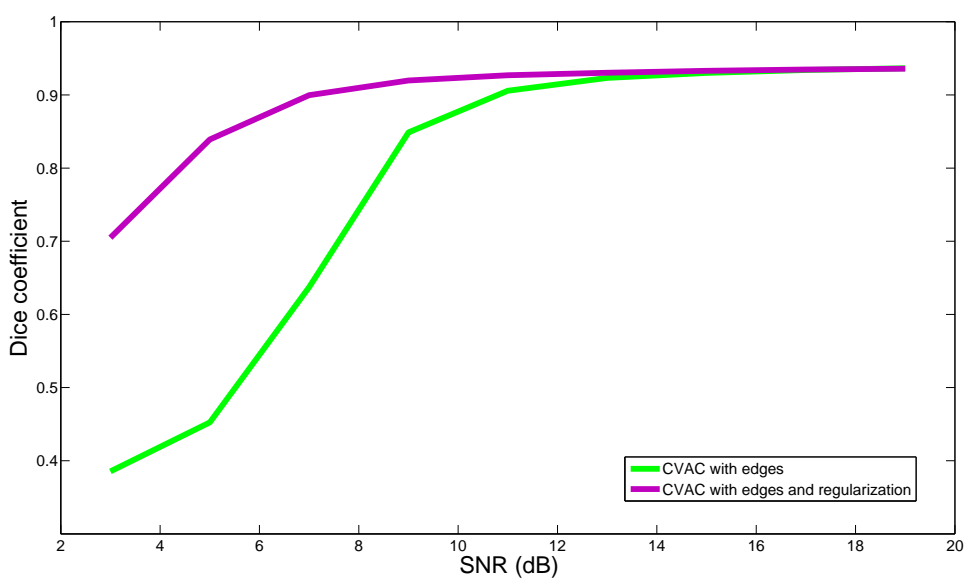

Fig. 5. The average Dice coefficient for segmentation of a synthetic dataset contaminated with different noise levels. The Dice coefficients were calculated for the segmentation result coming from the Chan-Vese active contours with edge information as for segmentation using the proposed method.

[13]. However it is experimentally proven that the proposed method is significantly more robust to noise and clutter than the method proposed in [13].

\section{Acknowledgment}

This research has been made possible by the Institute for the Promotion of Innovation by Science and Technology in Flanders (IWT).

\section{References}

1. Kass, M., Witkin, A., Terzopoulos, D.: Snakes: active contour models. International journal of computer vision (1988) 321-331

2. Isard, M., Blake, A.: Active contours. Springer (1998)

3. Ray, N., Acton, S.: Motion gradient vector flow: An external force for tracking rolling leukocytes with shape and size constrained active contours. IEEE transaction on medical imaging 23 (2004) 1466-1478

4. Tang, J.: A multi-direction gvf snake for the segmentation of skin cancer images. Pattern Recognition (2008)

5. Charmi, M.A., Derrode, S., Ghorbel, S.: Fourier-based geometric shape prior for snakes. Pattern Recognition Letters 29 (2008) 897-904

6. Rochery, M., Jermyn, I.H., Zerubia, J.: Higher order active contours. Int. J. Comput. Vision 69(1) (2006) 27-42

7. Chan, T., Vese, L.: An active contour model without edges. Scale-Space Theories in Computer Vision 1682 (1999) 141-151

8. Mille, J.: Narrow band region-based active contours and surfaces for $2 \mathrm{~d}$ and $3 \mathrm{~d}$ segmentation. Computer Vision and Image Understanding 113(9) (2009) 946-965 
9. Tsechpenakis, G., Rapantizikos, K., Tsapatsoulis, N., Kollias, S.: A snake model for object tracking in natural sequences. Signal processing: image communication 19 (2004) 219-238

10. Goldenberg, R., Kimmel, R., Rivlin, E., Rudzsky, M.: Fast geodesic active contours. IEEE Transactions on Image Processing 10(10) (2001) 1467-1475

11. Chan, T.F., Esedoglu, S., Nikolova, M.: Algorithms for finding global minimizers of image segmentation and denoising models. Siam Journal on Applied Mathematics 66(5) (2006) $1632-1648$

12. Goldstein, T., Bresson, X., Osher, S.: Geometric applications of the split bregman method: Segmentation and surface reconstruction. Journal of Scientific Computing 45(1-3) (2010) 272-293

13. Bresson, X., Esedoglu, S., Vandergheynst, P., Thiran, J.P., Osher, S.: Fast global minimization of the active contour/snake model. Journal of Mathematical Imaging and Vision 28(2) (2007) 151-167

14. Bresson, X., Chan, T.F.: Active contours based on chambolle's mean curvature motion. 2007 IEEE International Conference on Image Processing, Vols 1-7 (2007) 33-363371

15. Goldstein, T., Osher, S.: The split bregman method for 11-regularized problems. Siam Journal on Imaging Sciences 2(2) (2009) 323-343

16. Saad, Y.: Iterative methods for sparse linear systems. 2nd edn. SIAM, Philadelphia (Year)

17. Ruusuvuori, P., Lehmussola, A., Selinummi, J., Rajala, T., Huttunen, H., , Yli-Harja, O.: set of synthetic images for validating cell image analysis. Proc. of the 16th European Signal Processing Conference (EUSIPCO-2008) (2008)

18. Aleman-Flores, M., Alvarez, L., Caselles, V.: Texture-oriented anisotropic filtering and geodesic active contours in breast tumor ultrasound segmentation. Journal of Mathematical Imaging and Vision 28(1) (2007) 81-97 